\title{
FOTOSENSIBILIZACIÓN DE TiO 2 CON UN COLORANTE COMERCIAL PARA FOTODEGRADACIÓN DE CONTAMINANTES ORGÁNICOS EN AGUA
}

\section{$\mathrm{TiO}_{2}$ PHOTOSENSIBILIZATION WITH A COMMERCIAL DYE FOR PHOTODEGRADATION OF ORGANIC WATER POLLUTANTS}

\author{
Juliana Leguizamón ${ }^{1}$, César Quiñones ${ }^{2}$, Hilda Espinosa ${ }^{3}$, Víctor Sarria ${ }^{4}$
}

\begin{abstract}
${ }^{1}$ Ing. Químico. Grupo de Materiales Fotoactivos, Universidad de América. Bogotá, Colombia. julilegui@gmail.com, ${ }^{2}$ Dr. Sc. Universidad de Nacional, Bogotá, Colombia. cesarquinoness@unal.edu.co ${ }^{3}$ Ing. Químico. Grupo de Materiales Fotoactivos, Universidad de América. Bogotá, Colombia. Hilda.espinosa@profesores.edu.co, hilespq888@gmail.com, ${ }^{4}$ Ph.D. en Química. Departamento de Química, Universidad de Los Andes. Bogotá, Colombia vsarria@uniandes.edu.co
\end{abstract}

Rev. U.D.CA Act. E Div. Cient. 13 (2): 185-190, 2010

\section{RESUMEN}

En este estudio, se exploró la fotosensibilización del semiconductor $\mathrm{TiO}_{2}$, mediante un colorante comercial, con el objeto de aumentar sus propiedades catalíticas. Se evaluaron los siguientes parámetros: la concentración de colorante, el tiempo de sensibilización del semiconductor, la cantidad de moles de colorante adsorbidas sobre el semiconductor y el corrimiento de la absorción de la radiación del catalizador modificado hacia la región del espectro visible. Finalmente, se realizaron pruebas de la actividad fotocatalítica del catalizador modificado en la degradación del contaminante modelo (fenol). Se obtuvo una eficiencia de remoción de 33,85\% de la concentración inicial de fenol, comparada contra 18,98\% usando $\mathrm{TiO}_{2}$ sin modificar. La fotosensibilización del $\mathrm{TiO}_{2}$ con colorantes comerciales aparece como una alternativa técnica y económicamente viable, para incrementar las propiedades fotocatalíticas del $\mathrm{TiO}_{2}$.

Palabras clave: $\mathrm{TiO}_{2}$, sensibilización, ftalocianinas, azul directo 199.

\section{SUMMARY}

In this study, the photosensitization of $\mathrm{TiO}_{2}$ semiconductor with a commercial dye in order to increase its catalytic properties was explored. The following parameters were evaluated: the dye concentration used on the sensitization, the semiconductor sensitization time, the amount (unit: mol) of the dye, adsorbed on the semiconductor and the radiation absorption shift on the modified catalyst towards the visible spectrum region. Finally, the photocatalytic activity of the modified catalyst through the degradation of a pollutant model (phenol) was tested. A removal efficiency of 33.85 per cent of the initial concentration of phenol, compared to 18.98 per cent using unmodified $\mathrm{TiO}_{2}$ was obtained. Photosensitization of $\mathrm{TiO}_{2}$ with commercial dyes appears to be a technically and economically feasible alternative, which can be adopted as an option to increase the photocatalytic properties of $\mathrm{TiO}_{2}$.

Key words: $\mathrm{TiO}_{2}$, sensitization, phthalocyanines, direct blue 199 .

\section{INTRODUCCIÓN}

El dióxido de titanio $\left(\mathrm{TiO}_{2}\right)$, actualmente, es el material más utilizado para fotocatálisis en aplicaciones ambientales y un óxido metálico semiconductor con propiedades fotocatalíticas, cuando es excitado con luz ultravioleta (Rango de longitud de onda $=300-400 \mathrm{~nm}$ ) (Chatterjee, 2005). Cuando incide la radiación UV sobre una partícula de $\mathrm{TiO}_{2}$, los fotones de energía superior a la de su ancho de banda (diferencia energética entre las bandas de conducción y de valencia, Eg) son absorbidos por él mismo. Con una probabilidad bastante alta, estos fotones ceden su energía a los electrones situados en la banda de valencia (BV) y, una vez excitados, tienen energía cinética suficiente como para ocupar un estado energético de la banda de conducción (BC). De esta forma, se crean pares de electrón (e-) y hueco $\left(\mathrm{h}^{+}\right)$sobre la superficie del semiconductor, hallándose los primeros en la banda de conducción y los segundos en la banda de valencia (Chatterjee, 2005). 
Debido a que solo es activo en la región $\mathrm{UV}$, el $\mathrm{TiO}_{2}$ solo puede aprovechar alrededor de un $4 \%$ de la intensidad del espectro de luz solar (Chatterjee, 2005), lo cual, representa un limitante mayor, puesto que no se utilizan al máximo sus propiedades fotocatalíticas; sin embargo, una alternativa que viene siendo explorada para extender su rango de foto-respuesta a la región del visible es emplear compuestos fotosensibilizadores, tales como colorantes. Uno de los colorantes que puede constituir una alternativa es el azul directo $199\left(\mathrm{C}_{32} \mathrm{H}_{14} \mathrm{CuN}_{8} \mathrm{Na}_{2} \mathrm{O}_{6} \mathrm{~S}_{2}\right)$ (Figura 1), que pertenece a la familia química de las ftalocianinas.

Las ftalocianinas son sustancias químicas de colores intensos muy estables, no son tóxicas o contaminantes, presentan propiedades semiconductoras y tienen diversas aplicaciones en la industria, principalmente, en la producción de pigmentos (Garza \& Kharisov, 2008). El empleo de ftalocianinas para funciones relacionadas con fotocatálisis heterogénea en remediación ambiental ha sido estudiado por Iliev et al. (2003). Otros trabajos conectados con el uso de ftalocianinas para purificación de aguas son Chen et al. (2005); Zhiyu et al. (2006); Yu et al. (2003) y Kang et al. (2007). Se ha investigado acerca de estudios similares en recopilaciones bibliográficas especializadas (Blake, 2001).

En este artículo, se exploró el uso del DB199, para dopar el semiconductor $\mathrm{TiO}_{2}$, con el ánimo de extender el rango de foto-respuesta a la región del visible y mejorar su actividad fotocatalítica.

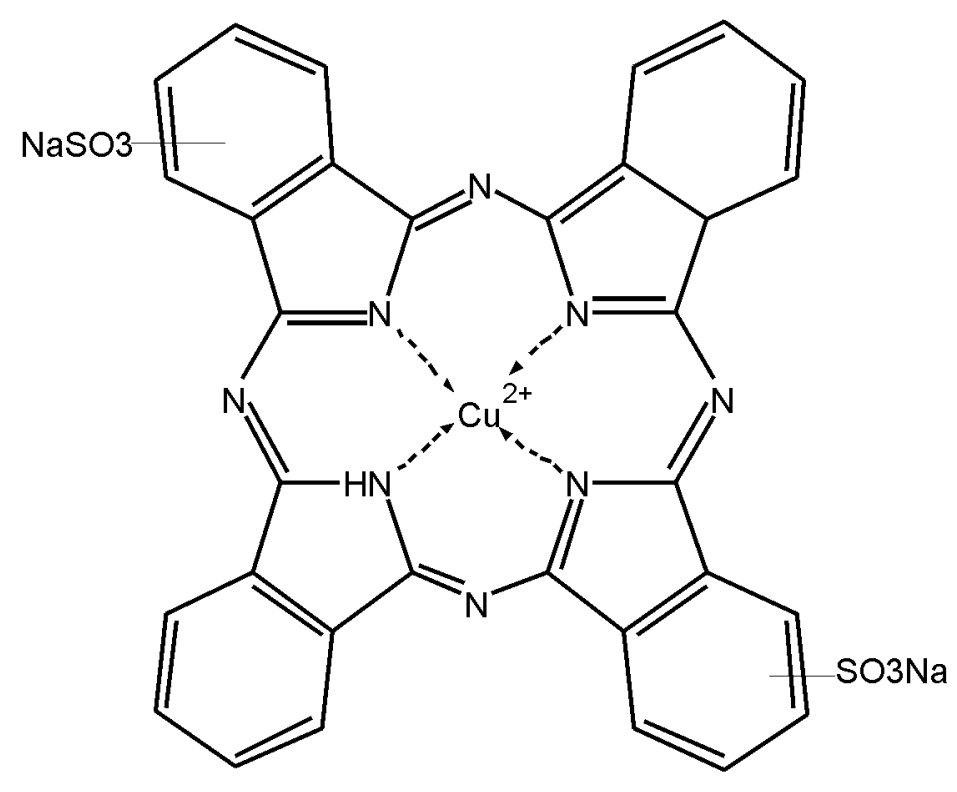

Figura 1. Estructura química del colorante Azul directo 199 (Turquesa Novazol JRLH Conc).

\section{MATERIALES Y MÉTODOS}

Preparación del fotocatalizador: La selección de la concentración de DB199 para hacer las pruebas de fotosensibilización, se realizó mediante revisión bibliográfica (El Mekkawi et al. 2005); los rangos de concentración manejados oscilan entre $1 \times 10^{-4} \mathrm{M}$ y $1 \times 10^{-5} \mathrm{M}$.

Se utilizaron 4mL de una solución de DB199 6.25X10-5 M y $0,02 \mathrm{~g}$ de $\mathrm{TiO}_{2}$ Degussa P25 $\left(\mathrm{TiO}_{2}\right)$. Para hacer el seguimiento de la concentración del DB199 y también de la degradación de fenol, se utilizó un espectrofotómetro GENESYS 20, leyendo a longitudes de onda de $\lambda_{\max }=622 \mathrm{~nm}$ y $\lambda_{\max }=275 \mathrm{~nm}$, respetivamente.
Tiempo de sensibilización: La cantidad de colorante adsorbido sobre el $\mathrm{TiO}_{2}$ es proporcional al tiempo de sensibilización (Yu et al. 2003). Se hizo seguimiento a la concentración adsorbida, mediante mediciones espectrofotométricas del filtrado del $\mathrm{TiO}_{2}$, sensibilizado en intervalos de una hora, durante seis horas. Se calcularon los moles de colorantes adsorbidos según Bauer et al. (1999).

Evaluación fotocatalítica: Se manipuló un reactor fotocatalítico, donde se evaluó la degradación del contaminante modelo (Fenol). El foto-reactor consta de un cuerpo fabricado en aluminio de $32 \mathrm{~cm} \times 30 \mathrm{~cm} \times 72 \mathrm{~cm}$, con una puerta frontal, cinco lámparas de luz blanca (20W) y tres agitadores magnéticos. Se usaron 50,100 y 150mg/L de cantidad de 
catalizador. Se degradaron $50 \mathrm{~mL}$ de fenol a $10 \mathrm{mg} / \mathrm{L} \mathrm{El} \mathrm{pH}$ del medio fue alcalino $(\mathrm{pH}=8)$, porque favorece la acción fotocatalítica de la ftalocianina sobre el $\mathrm{TiO}_{2}$ (Ozoemena et al. 2001). La degradación, se llevó a cabo a temperatura ambiente, dado que la activación fotónica del catalizador no requiere aporte de temperatura, puesto que la energía térmica no produce excitación sobre el catalizador (Fernández, 2004).

La evolución de la concentración de fenol, se efectuó mediante espectrofotometría UV- Visible, retirando el catalizador, a través de filtración; los porcentajes de degradación, se calcularon utilizando la ecuación 1 :

$$
\% D=\left\lfloor\frac{\left(C_{i}-C_{F}\right)}{C_{i}}\right\rfloor * 100 \%
$$

Ecuación 1 (Zhiyu et al. 2006)

Donde:

\%D: Porcentaje de degradación de fenol.

$\mathrm{C}_{\mathrm{i}}$ : Concentración inicial de fenol (mg/L).

$\mathrm{C}_{\mathrm{f}}$ : Concentración final de fenol (mg/L).

\section{RESULTADOS Y DISCUSIÓN}

\section{Fotosensibilización de $\mathrm{TiO}_{2}$ con DB199}

La figura 2 muestra la variación de la cantidad de moles adsorbidos (mol DB199/g TiO $)$ frente a la variación de tiempo de sensibilización $(t=1-6 h)$. Se encontró que en dos horas se fijó 12,2 mol x10-6 $\mathrm{mol} \mathrm{DB} 199 / \mathrm{g} \mathrm{TiO}_{2}$, siendo este periodo el utilizado para modificar el $\mathrm{TiO}_{2}$ empleado en la degradación del contaminante. Si bien es cierto que los moles adsorbidos en el $\mathrm{TiO}_{2}$ son directamente proporcionales al tiempo, en el proceso, llega un momento donde la superficie del $\mathrm{TiO}_{2}$ adsorbe una cantidad límite de DB199.

Para determinar la adsorción del DB199 sobre el $\mathrm{TiO}_{2}$, se recurrió a los resultados obtenidos en las pruebas de tiempo de sensibilización. Este estudio, se basó en el trabajo de Bauer et al. (1999), donde se afirma que la adsorción de compuestos químicos en los semiconductores, sigue el modelo de la isoterma de Langmuir.

En la adsorción del colorante sobre el $\mathrm{TiO}_{2}$ existe competencia entre el soluto (DB199) y solvente (etanol), para ocupar sitios en la superficie del catalizador $\left(\mathrm{TiO}_{2}\right)$; por lo cual, el sistema se trata como una reacción de adsorción - desorción superficial. La isoterma de Langmuir fue construida según (Bauer et al. 1999).

En la figura 3, se presenta la isoterma de Langmuir, construida a partir de la ecuación 2 , que muestra formación de monocapa del DB199 sobre el $\mathrm{TiO}_{2}\left(\mathrm{y}=0.0981 \mathrm{x}-0.4579 ; \mathrm{R}^{2}=0.9976\right)$ y que la cantidad máxima de colorante adsorbida sobre el semiconductor es $10.19 \times 10^{-6} \mathrm{~mol} \mathrm{DB} 199 / \mathrm{g} \mathrm{TiO}_{2}$.

$$
\frac{C_{e q}}{n_{a d s}}=\frac{1}{K n_{\max }}+\frac{C_{e q}}{n_{\max }}
$$

Ecuación 2 (Bauer et al. 1999)

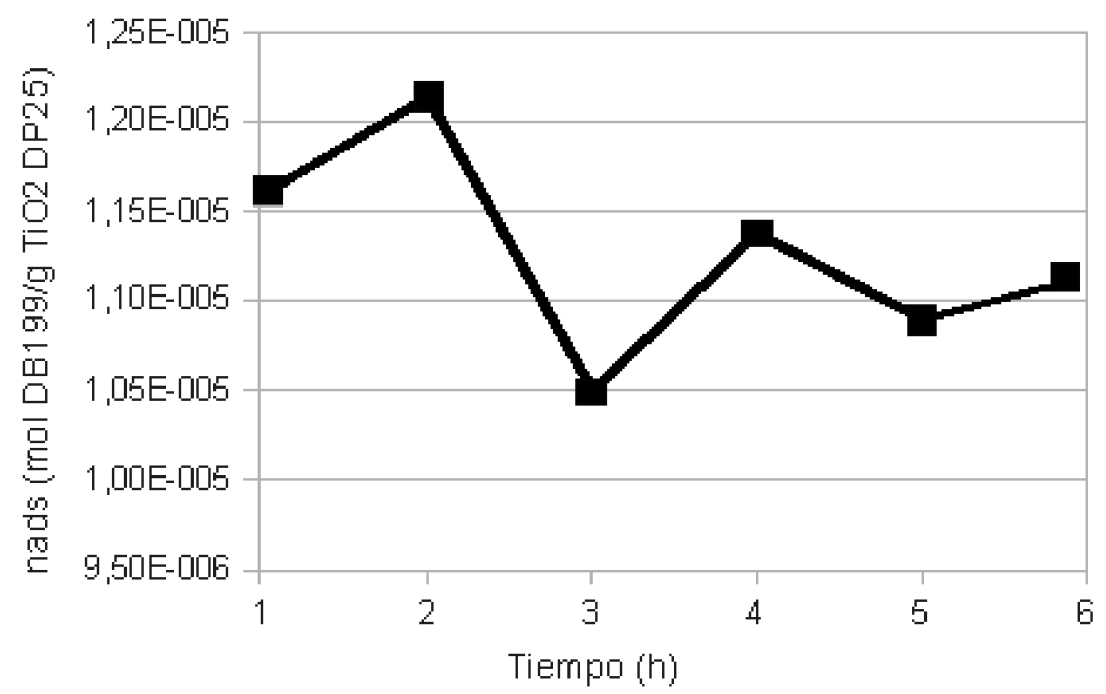

Figura 2. Variación de moles adsorbidos de DB199 sobre el TiO frente al tiempo de sensibilización. 
Donde:

Ceq $=$ Concentración de DB199 en el equilibrio (M)

$\mathrm{n}_{\mathrm{ads}}=$ Moles de DB199 adsorbidos (Mol DB199/g TiO ${ }_{2}$ )

$\mathrm{n}_{\max }=$ número total de sitios de adsorción

$\mathrm{K}=$ constante de asociación $\left(M^{-1}\right)$

Debido a que los tiempos de adsorción obtenidos se ajustaron a la ecuación 2, se determinó que el DB199 fue adsorbido sobre la superficie del $\mathrm{TiO}_{2}$ en forma de monocapa, ajustándose al modelo de adsorción - desorción de la isoterma de Langmuir, donde el soluto (colorante) y el solvente (etanol) compiten entre sí, para ocupar sitios en la superficie del catalizador. Se sugiere en un próximo trabajo realizar mediciones que permitan confirmar y precisar la forma de anclaje del colorante sobre el catalizador.
Para conocer en cuánto se extendió el rango de fotorespuesta del semiconductor, se procedió a una medición de transmitancia del $\mathrm{TiO}_{2}$ y del $\mathrm{TiO}_{2}-\mathrm{DB} 199$. En la figura 4 , se muestran los espectros del $\mathrm{TiO}_{2}$ y el $\mathrm{TiO}_{2}-\mathrm{DB} 199$. Se puede observar que hubo una disminución en la transmitancia del $\mathrm{TiO}_{2}$ - DB199 proporcional al aumento de longitud de onda. Dicho fenómeno, se puede interpretar como un incremento de absorción de luz visible por parte del $\mathrm{TiO}_{2}$, consecuencia de la presencia del DB199 en la superficie del semiconductor. El incremento fue desde $\lambda=400 \mathrm{~nm}$ a $\lambda=$ $460 \mathrm{~nm}$ aproximadamente, donde la última es la longitud de onda de absorción característica del color azul. Los resultados obtenidos están de acuerdo con las lecturas de transmitancia y pruebas de reflectancia difusa de investigaciones, donde se utilizaron complejos ftalocianinicos en la degradación de contaminantes y la caracterización espectral de dichos materiales según Yu et al. (2003); Kumar et al. (2003); Ding et al. (2005); Zhiyu et al. (2006) y Kang et al. (2007).

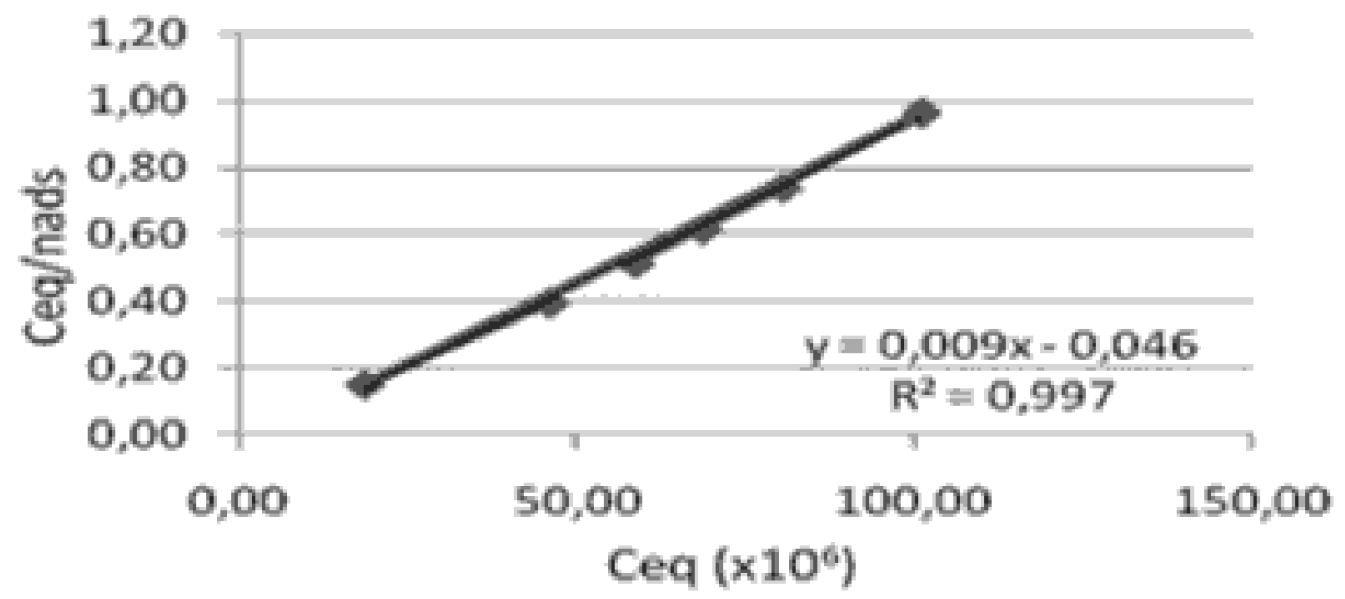

Figura 3. Isoterma de Langmuir del $\mathrm{TiO}_{2}-\mathrm{DB} 199$.

\section{Evaluación fotocatalítica del $\mathrm{TiO}_{2}$ fotosensibilizador}

La figura 5 muestra los resultados experimentales de la degradación de fenol (contaminante modelo) durante seis horas, comparando la eficiencia del catalizador $\mathrm{TiO}_{2}$ y el $\mathrm{TiO}_{2}$-DB199, usando diferentes cantidades de catalizador. No se observaron cambios en la concentración inicial del contaminante antes de comenzar la irradiación con luz visible. Durante las pruebas, el $\mathrm{TiO}_{2}$ presentó una baja eficiencia de degradación. Se puede advertir, que el mayor porcentaje de degradación obtenido fue de $18,98 \%$, correspondiente a una concentración de catalizador de $150 \mathrm{mg} / \mathrm{L}$. Cuando se usaron $100 \mathrm{mg} / \mathrm{L}$ de $\mathrm{TiO}_{2}-\mathrm{DB} 199$, se logró la mayor disminución del contaminante, $33,85 \%$, lo que se puede atribuir a que a esta concentración de catalizador se absorbió la mayor proporción de fotones. Si se supera este límite, se disminuye el rendimiento de la degradación del contaminante, posiblemente, a reacciones de competición y también al efecto de pantalla que puede ejercer el exceso de $\mathrm{TiO}_{2}$.

Fue viable degradar fenol con el semiconductor modificado, bajo irradiación continua con luz visible, durante seis horas.

Se logró sensibilizar el $\mathrm{TiO}_{2}$ con el colorante comercial DB199. Se necesitaron dos horas para obtener la mayor cantidad de moles de colorante adsorbidas sobre la superficie del catalizador. El colorante provocó el corrimiento de la banda de absorción del $\mathrm{TiO}_{2}$ hacia la región del espectro visible, 


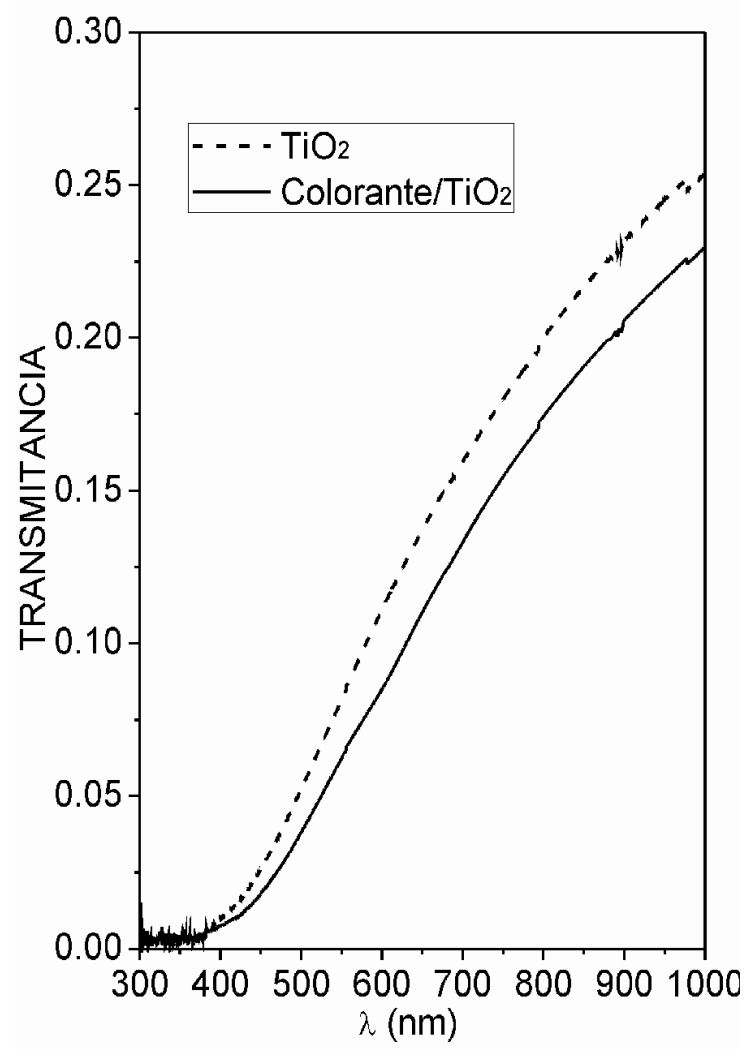

Figura 4. Variación de la transmitancia frente a la Longitud de Onda del $\mathrm{TiO}_{2}\left(\mathrm{TiO}_{2}\right)$ y del $\mathrm{TiO}_{2}-\mathrm{DB} 199\left(\right.$ Colorante - $\left.\mathrm{TiO}_{2}\right)$.

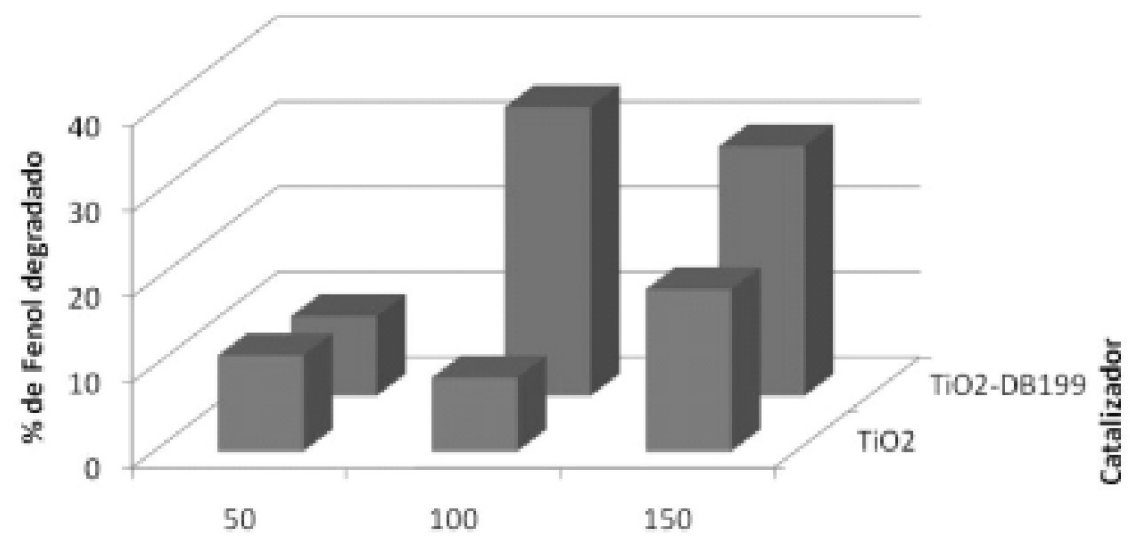

Cantidad de catalizador $\left(\mathrm{mg} \mathrm{L}^{-1}\right)$

Figura 5. Comparación de la eficiencia fotocatalítica del $\mathrm{TiO}_{2}$ y $\mathrm{TiO}_{2}$-DB199, a diferentes cantidades, en la degradación de fenol. 
lo que abre la posibilidad de usar este nuevo material bajo irradiación con luz solar. Usando el $\mathrm{TiO}_{2}$-DB199 fue factible degradar aproximadamente $34 \%$ de la concentración inicial del contaminante modelo fenol en el reactor fotocatalítico evaluado. En general, la alternativa de fotosensibilización empleando colorantes comerciales aparece como una alternativa promisoria en busca de alternativas ambientalmente amigables, para la remediación ambiental.

Agradecimientos. La autora principal agradece a los co-autores de este artículo por todo su interés, apoyo y disposición de los medios necesarios para el desarrollo conceptual y experimental de este proyecto de investigación. Financiación: Este trabajo fue realizado gracias a las donaciones de materiales realizadas por Colorquimica S.A., Grupo Alianza Estrategica (GAE) S.A. Se agradece el apoyo económico del Fondo Semilla de la Facultad de Ciencias de la Universidad de los Andes 2010. Conflictos de Intereses: Este manuscrito fue preparado y revisado por todos los autores, quienes declaramos que no existe ningún conflicto de intereses que ponga en riesgo la validez de los resultados presentados.

\section{BIBLIOGRAFÍA}

1. BAUER, C.; JACQUES, P.; KALT, A. 1999. Investigation of the interaction between a sulfonated azo dye (AO7) and a $\mathrm{TiO}_{2}$ surface. Chemical Physics Letters. 307:397-406.

2. BLAKE, D. 2001. Bibliography of work on the heterogeneus photocatalytic removal of hazardous compounds from water and air. NREL/TP - 510 - 31319. Update \# 4 to October 2001 Disponible desde Internet en www.nrel. gov/docs/fy02osti/31319.pdf (con acceso: 10/05/10).

3. CHATTERJEE, D.; DASGUPTA, S. 2005. Visible light induced photocatalytic degradation of organic pollutants. J. Photochem. Photobiol. C: Photochemistry Reviews. 6:186-205.

4. CHEN, F.; DENG, Z.; LI, X.; ZHANG, J.; ZHAO, J. 2005. Visible light detoxification by 2,9,16,23-tetracarboxyl phthalocyanine copper modified amorphous titania. Chem. Phys. Letters 415:85-88.

5. DING, H.; ZHANG, X.; KUMAR, R.M.; NICOLINI, C. 2005. Ultrathin films of tetrasulfonated copper phthalocyanine-capped titanium dioxide nanoparticles: Fabrication, characterization, and photovoltaic effect. Journal of Colloid and Interface Science 290:166-171.

6. EL MEKKAWI, D.; ABDEL-MOTTALEB, M.S.A. 2005. The interaction and photostability of some xanthenes and selected azo sensitizing dyes with $\mathrm{TiO}_{2}$ nanoparticles. Internal J. Photoenergy. 7:95-101.

7. FERNÁNDEZ, P. 2004. Propiedades coloidales de partículas de $\mathrm{TiO}_{2}$ : Aplicación al tratamiento fotocatalítico solar de aguas. U. de Almería (Dpto. de Física Aplicada), Plataforma solar de Almería - CIEMAT (Ministerio de Ciencia y Tecnología). p.280-283.

8. GARZA, L.A.; KHARISOV, B. 2008. Pigmentos tipo Ftalocianinas no sustituidas: Obtención y propiedades. Facultad de Ciencias Químicas - UANL. Revista Ingenierías. 11(38):43-51.

9. ILIEV, V.; TOMOVA, D.; BILYARSKA, L.; PRAHOV, L.; PETROV, L. 2003. Phthalocyanine modified $\mathrm{TiO}_{2}$ or $\mathrm{WO}_{3}-$ catalysts for photooxidation of sulfide and thiosulfate ions upon irradiation with visible light. J. Photochemistry and Photobiology A: Chemistry 159:281-287.

10. KANG, C.; LU, Y.; GUO, P.; ZHANG, F.; YU, H.; LIN, X. 2007. Photooxidation of Furfural with phthalocyanine sensitized $-\mathrm{TiO}_{2}$ particle under Xenon lamp. Chem. Res. Chinese U. 23(2):154-158.

11. KUMAR, G.A.; GIN, J.; VINOY, T.; UNNIKRISHNAN, N.V.; NAMPOORI, V.P.N. 2003. NIR to UV absorption spectra and the optical constants of phthalocyanines in glassy medium. Spectrochimica Acta Part A. 59:1-11.

12. OZOEMENA, K.; KUTZNETSOVA, N.; NYOKONG, T. 2001. Photosensitized transformation of 4 - chlorophenol in the presence of aggregated and not - aggregated metallophthalocyanines. J. Photochem. Photobiology A. Chemistry 139:217-224.

13. YU, J.C.; XIE, Y.; TANG, H.Y.; ZHANG, L.; CHAN H.C.; ZHAO, J. 2003. Visible Light - assisted bacterial effect of metalphthalocyanine - sensitized titanium dioxide films. J. Photochem. Photobiol. A: Chemistry 156:235241.

14. ZHIYU, W.; HAIFENG, C.; PEISONG, T.; WEIPING, M.; FUAN, Z.; GUODONG, Q.; XIANPING, F. 2006. Hydrothermal in situ preparation of the copper phthalocyanine tetrasulfonate modified titanium dioxide photocatalyst. Colloids and Surfaces A: Physicochem. Eng. Aspects 289:207-211.

Recibido: Noviembre 30 de 2009

Aceptado: Octubre 22 de 2010 